\title{
Education of Nanoscience: Introduction to the Preparation, Characterization, and Application of Gold Nanoparticles
}

\author{
Yang-Wei Lin \\ Department of Chemistry/ National Changhua University of Education \\ 1, Jin-De Road, Changhua City, Taiwan \\ linywjerry@cc.ncue.edu.tw
}

\section{Extended Abstract}

Objectives: We demonstrate a simple method for the synthesis of gold nanoparticles (Au NPs) via a boiling and refluxing methodology [1]. The proposed experiment also can help students to understand the factors involved in the stability of Au NPs by exploring the adsorption interaction between Au NPs and various substances [2].

Scope: The students in this study found that the surface plasmon resonance band of Au NP solutions underwent a redshift (i.e., from 520 to $650 \mathrm{~nm}$ ) because of $\mathrm{NaCl}$-induced aggregation caused by the elimination of the repulsive electrostatic force. In addition, a sufficient amount of bovine serum albumin molecules adsorbed on the surface of Au NPs through electrostatic interactions provides steric barriers that hinder electrolyte-induced aggregation. The surface properties of $\mathrm{Au}$ NPs are important in determining the aggregation of Au NPs. An optical sensor for $\mathrm{Hg}^{2+}$ ions is developed by using 3-MPA-modified Au NPs (3-MPA-Au NPs) [3, 4]. If their aggregation was driven by the recognition and binding of metal ions, the color change would allow sensing of the ions by the naked eye. The experiment possesses several attractive features: the synthesis method circumvents the use of a surfactant or template; the selectivity and sensitivity experiments use small amounts of reagents; and the whole experiment can be carried out within $3 \mathrm{hrs}$. at room temperature.

Results: In addition, this current teaching material also provides students with an introduction to major concepts of nanoscale science and engineering [5] including (i) size and scale, (ii) structure of matter, (iii) forces and interactions, (iv) self-assembly, (v) tools and instrumentation, and (vi) science, technology, and society. Hence, these teaching materials are suitable for incorporation into the undergraduate general chemistry laboratory curriculum. Students performed this experiment and improved their recognition of the nanoscale science and engineering concepts.

Keywords: General Chemistry, Laboratory Instruction, Curriculum, Hands-On Learning, Nanotechnology

\section{References}

[1] Y.-W. Lin, P.-Y. Cheng, J.-C. Tsai, C.-F. Li, C.-H. Chou, P.-Y. You, N.-Y. Hsu, C.-I Wang, and C.-L. Lai, “A Colorimetric Sensing of $\mathrm{Hg}$ (II) Ions Using 3-Mercaptopropionic Acid Modified Au Nanoparticles for the Undergraduate Chemistry Laboratory Curriculum,” J. Nano Edu., vol. 7, pp. 1-9, 2015.

[2] C.-F. Lee, P.-Y. You, Y.-C. Lin, T.-L. Hsu, P.-Y. Cheng, Y.-X. Wu, C.-S. Tseng, S.-W. Chen, H.-P. Chang, and Y.-W. Lin, "Exploring the Stability of Gold Nanoparticles by Experimenting with Adsorption Interactions of Nanomaterials in an Undergraduate Lab," J. Chem. Edu., vol. 92, pp. 1066-1070, 2015.

[3] Y. -W. Lin, W. -T. Chen, and H. -T. Chang, "Exploring the Interactions between Gold Nanoparticles and Analytes through Surface-Assisted Laser Desorption/Ionization Mass Spectrometry," Rapid Commun. Mass Spectrom., vol. 24, pp. 933-938, 2010.

[4] C. -C. Huang and H. -T. Chang, "Parameters for selective colorimetric sensing of mercury(II) in aqueous solutions using mercaptopropionic acid-modified gold nanoparticles," Chem. Commun., vol. 12, pp. 1215-1217, 2007.

[5] S. Y. Stevens, L. M. Sutherland, and J. S. Krajcik, The big ideas of nanoscale science and engineering. Arlington, VA: NSTA Press, 2009. 